\title{
Optical and Structural Characterization of Zinc Oxide Nanostructures Obtained by Atomic Layer Deposition Method
}

\author{
Ł. Wachnicki ${ }^{a}$, B.S. Witkowski ${ }^{a}$, S. Gieraetowska ${ }^{a}$, K. Kopalko $^{a}$, \\ M. GODLEWSKI ${ }^{a, b}$ AND E. GUZIEWICZ ${ }^{a}$ \\ ${ }^{a}$ Polish Academy of Sciences, Institute of Physics, al. Lotników 32/46, 02-668 Warsaw, Poland \\ ${ }^{b}$ Cardinal Stefan Wyszyński University, College of Science, Department of Mathematics \\ and Natural Sciences, Warsaw, Poland
}

\begin{abstract}
Zinc oxide is a II-VI semiconductor material which is gaining increasing interest in various fields such as biology, medicine or electronics. This semiconductor reveals very special physical and chemical properties, which imply many applications including a transparent electrode in solar cells or LED diodes. Among many applications, $\mathrm{ZnO}$ is also a prospective material for sensor technology, where developed surface morphology is very advantageous. In this work we present $\mathrm{ZnO}$ nanowires growth using atomic layer deposition method. $\mathrm{ZnO}$ nanowires were obtained using controlled physical properties. As a substrate we used gallium arsenide with gold-gallium eutectic droplets prepared on the surface at high temperature. To obtain the eutectic solution there was put a gold thin film on GaAs through the sputtering and then we annealed the sample in a nitrogen gas flow. The so-prepared substrate was applied for growth of $\mathrm{ZnO}$ nanowires. We used deionized water and zinc chloride as oxygen and zinc precursors, respectively. The eutectic mixture serves as a catalyst for the $\mathrm{ZnO}$ nanowires growth. $\mathrm{Au}-\mathrm{Ga}$ droplets flow on the front of $\mathrm{ZnO}$ nanowires. Scanning electron microscopy images show $\mathrm{ZnO}$ nanorods in a form of crystallites of up to $1 \mu \mathrm{m}$ length and a $100 \mathrm{~nm}$ diameter. It is the first demonstration of the $\mathrm{ZnO}$ nanowires growth by atomic layer deposition using the vapour-liquid-solid approach.
\end{abstract}

PACS: 81.07.Bc, 82.47.Rs, 68.55.-a, 81.15.Aa

\section{Introduction}

The atomic layer deposition (ALD) method was developed by Suntola and originally was used for epitaxial growth [1]. The simplicity of this method causes that people used ALD technique not only for the single crystal deposition, but also for growth of polycrystalline and amorphous layers [2-4]. In this paper we present nanostructure of zinc oxide which is a II-VI semiconductor with the direct band gap energy of $3.37 \mathrm{eV}$ [5] at room temperature. It has a high exciton binding energy $(60 \mathrm{meV})[6]$, which have a large influence on the optical properties of the material. Simultaneously, $\mathrm{ZnO}$ has a very high chemical stability, which makes it a good candidate as devices component.

Due to very interesting properties zinc oxide has many electronics applications. The wide energy gap of this material makes it transparent in the visible range of the electromagnetic spectrum. ZnO strongly doped with aluminum can be used as a transparent conductive oxide (TCO). Due to the high exciton binding energy, it is possible to use $\mathrm{ZnO}$ in the light emitting diode (LED) [7].

The protection of the natural environment is very important due to the rapid development of industry. The essential key of environmental protection is to monitor changes in the atmosphere and hydrosphere. Therefore, construction of sensors that detects the composition of gas in the atmosphere is crucial for industry. Different gases or liquids have a very strong influence on the conductivity of the $\mathrm{ZnO}$ surface, thus selection of $\mathrm{ZnO}$ for sensor applications was obvious. In this paper we present two methods of preparing such sensors based on $\mathrm{ZnO}$, by using the ALD method.

\section{Experimental details}

Atomic layer deposition processes were performed on gallium arsenide substrates. Cleaning chemical processes like etching and rinsing in solvents were used prior to the $\mathrm{ZnO}$ deposition. After chemical preparation, a thin layer of gold was deposited. The substrate with a gold layer was annealed in the rapid thermal process (RTP). In this process we obtained $\mathrm{Au}-\mathrm{Ga}$ eutectic in form of droplets (shown in Fig. 1). Diameter of a single droplet was about $100 \mathrm{~nm}$. After processing, substrates were placed in the ALD reactor - Microchemistry F-120 ALD system.

Zinc oxide nanostructures were deposited in a double exchange reaction using zinc chloride and deionized water as precursors 


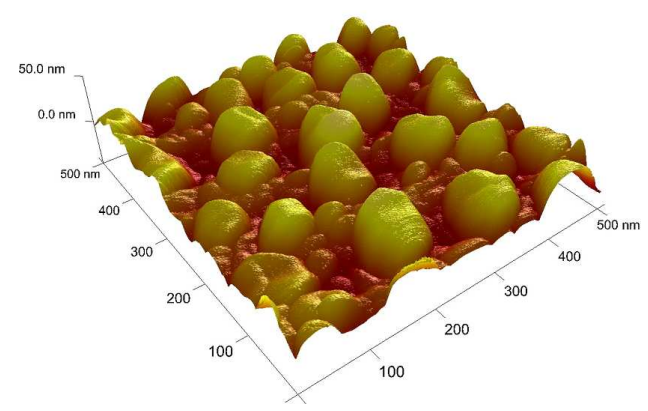

Fig. 1. Image of eutectic droplets $\mathrm{Ga}-\mathrm{Au}$ observed by AFM.

$$
\mathrm{ZnCl}_{2}+\mathrm{H}_{2} \mathrm{O} \rightarrow \mathrm{ZnO}+2 \mathrm{HCl}
$$

Between pulses of precursors, the reaction chamber was purged by high purity nitrogen gas (99.9999\%). Optical properties of zinc oxide nanostructures have been characterized by the spectrofluorimeter CM2203, where the excitation source was the xenon lamp. Surface morphology was investigated by atomic force microscope (AFM) and scanning electron microscope (SEM - Hitachi SU-70).

\section{Result and discussion}

As a result of optimization of the growth process, we received two types of zinc oxide nanostructures (technical name: Nano1, see Fig. 2a, and Nano2 - Fig. 2b). The Nano1 structure was characterized by a columnar growth of zinc oxide. The typical nanowire size was about $1 \mu \mathrm{m}$ length, while the diameter was about $250 \mathrm{~nm}$. Such nanostructures grew directly on $\mathrm{Ga}-\mathrm{Au}$ droplets. These droplets nucleated growth of zinc oxide nanowires. Zinc oxide nanocolumns growth on a pure gallium arsenide substrate (without $\mathrm{Ga}-\mathrm{Au}$ droplets) was not observed. The structure of Nano2 was also characterized by a columnar growth, but in this case the growth of $\mathrm{ZnO}$ nanostructures was catalyzed by a $\mathrm{Ga}-\mathrm{Au}$ droplet, which floats on the front of growth and freezes at the top after the process. Thus, this is a typical vapor-liquid-solid (VLS) growth mode. The size of the single nanowire of Nano2 structure was about $1 \mu \mathrm{m}$ length and the diameter of about $50 \mathrm{~nm}$.

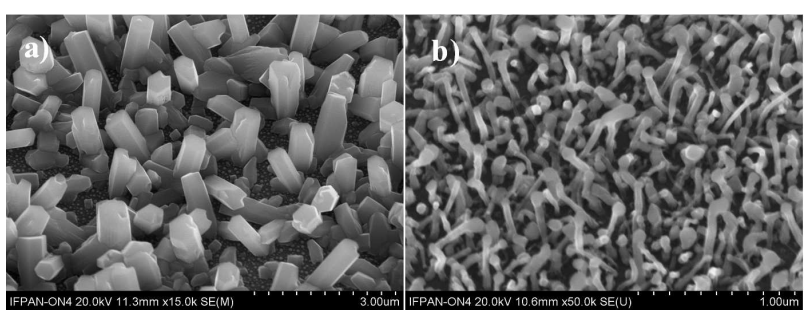

Fig. 2. Surface structure of (a) Nano1, (b) Nano2 characterized by SEM.

Figure 3 shows the photoluminescence intensity as a function of wavelength for the two nanostructures. For
Nano1 we observed a strong "edge" luminescence (around $380 \mathrm{~nm}$ ). High intensity photoluminescence in this area confirms good quality of obtained zinc oxide nanostructures. In the wavelength range from $450 \mathrm{~nm}$ to $700 \mathrm{~nm}$ we observed a very weak (in comparison to "edge luminescence") defect-related luminescence. For Nano2 nanostructure "edge", defect-related luminescence was very weak or even not observed. Probably, the intensity of photoluminescence depends of zinc oxide crystal size.

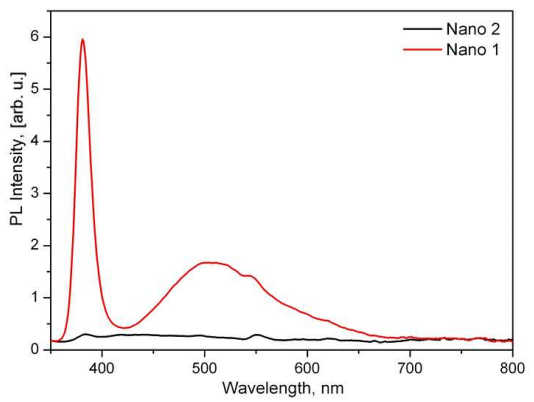

Fig. 3. Room temperature photoluminescence of Nano1 and Nano2 nanostructures.

Nano1 and Nano2 structures were tested for sensor application. We deposited $\mathrm{Ti} / \mathrm{Au}$ ohmic contacts on their surface, and performed time--resistance measurements.

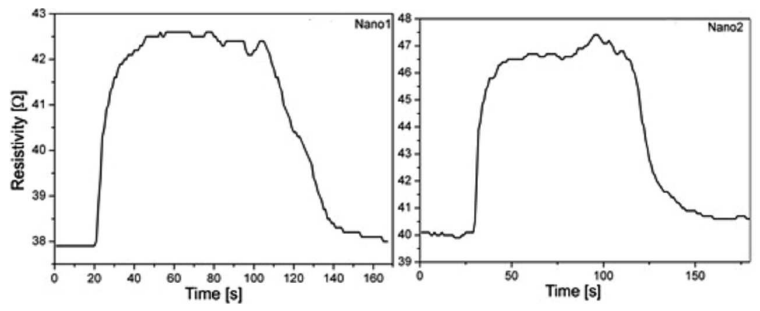

Fig. 4. Dependence of resistivity of Nano1 and Nano2 nanostructures after applying a drop of acetone.

Samples resistance was tested with different solvents like acetone, isopropanol, trichloroethylene and ethanol deposited on the surfaces. Figure 4 presents a graph showing the sensor behavior for two obtained structures (Nano1 and Nano2). After deposition of the acetone droplet on the sample surface, resistance increased significantly. After evaporation of solvent from the surface, measured resistance returns to the state before the drop deposition. A similar result was observed for all solvents but magnitude of the response was solvent dependent. We observed a very advantageous property of obtained nanosensors. Sensors were reset to their initial resistivity within a few minutes without any surface treatment or heating. If heating was applied, the resetting time was significantly shorter. We observed an increase of resistivity for several tested solvents and alcohols. The problem to be solved is now a selectivity of the response to different chemical compounds. 


\section{Conclusions}

Zinc oxide nanostructures were performed by the atomic layer deposition process. The so-obtained nanostructures show high sensitivity to chemical solvents and alcohols. The sensors structure can be applied for detection of hazardous substances in a natural environment. Importantly, these devices were automatically reset after solvent or alcohol evaporation, without any annealing and chemical processing.

Very important is also the method of obtaining zinc oxide nanostructures, which can be easily implemented in the industry. Our technology of production the nanowires are relatively a cheap method and seem to be a good alternative against other methods of growing such nanostructures.

\section{Acknowledgments}

The research was supported by the European Union within European Regional Development Fund, through grant Innovative Economy (POIG.01.01.02-00-008/08).

\section{References}

[1] T. Suntola, in: Handbook of Crystal Growth, Part 3b: Growth Mechanisms and Dynamics, Ed. D.T.J. Hurle, Elsevier, Amsterdam 1994, p. 605.

[2] Ł. Wachnicki, T. Krajewski, G. Łuka, B. Witkowski, B. Kowalski, K. Kopalko, J.Z. Domagala, M. Guziewicz, M. Godlewski, E. Guziewicz, Thin Solid Films 518, 4556 (2010).

[3] E. Przezdziecka, T. Krajewski, L. Wachnicki, A. Szczepanik, A. Wójcik-Głodowska, S. Yatsunenko, E. Lusakowska, W. Paszkowicz, E. Guziewicz, M. Godlewski, Acta Phys. Pol. A 114, 1303 (2008).

[4] S. Gierałtowska, D. Sztenkiel, E. Guziewicz, M. Godlewski, G. Łuka, B.S. Witkowski, Ł. Wachnicki, E. Łusakowska, T. Dietland, M. Sawicki, Acta Phys. Pol. A 119, 692 (2011).

[5] C. Klingshirn, Phys. Status Solidi B 244, 3027 (2007).

[6] S.J. Pearton, D.P. Norton, K. Ip, Y.W. Heo, T. Steiner, Superlattices Microstruct. 34, 3 (2003).

[7] M. Godlewski, E. Guziewicz, G. Łuka, T. Krajewski, M. Łukasiewicz, Ł. Wachnicki, A. Wachnicka, K. Kopalko, A. Sarem, B. Dalami, Thin Solid Films 518, 1145 (2009). 